\title{
Acoustic multipole source model for volcanic explosions and inversion for source parameters
}

\author{
Keehoon Kim, ${ }^{1}$ Jonathan M. Lees ${ }^{1}$ and Mario Ruiz ${ }^{2}$ \\ ${ }^{1}$ Department of Geological Sciences, University of North Carolina at Chapel Hill, Chapel Hill, NC, USA. E-mail: keehoon.kim@gmail.com \\ ${ }^{2}$ Instituto Geofísico - Escuela Politécnica Nacional (IGEPN) Ladrón de Guevara E11-253. Quito, Ecuador
}

Accepted 2012 September 25. Received 2012 September 12; in original form 2011 November 7

\begin{abstract}
S UMMAR Y
Volcanic explosions are accompanied by strong acoustic pressure disturbances in the atmosphere. With a proper source model, these acoustic signals provide invaluable information about volcanic explosion dynamics. Far-field solutions to volcanic infrasound radiation have been derived above a rigid half-space boundary, and a simple inversion method was developed based on the half-space model. Acoustic monopole and dipole sources were estimated simultaneously from infrasound waveforms. Stability of the inversion procedure was assessed in terms of variances of source parameters, and the procedure was reliable with at least three stations around the infrasound source. Application of this method to infrasound observations recorded at Tungurahua volcano in Ecuador successfully produced a reasonable range of source parameters with acceptable variances. Observed strong directivity of infrasound radiation from explosions at Tungurahua are successfully explained by the directivity of a dipole source model. The resultant dipole axis, in turn, shows good agreement with the opening direction of the vent at Tungurahua, which is considered to be the origin of the dipole source. The method is general and can be utilized to study any monopole, dipole or combined sources generated by explosions.
\end{abstract}

Key words: Inverse theory; Acoustic properties; Volcano monitoring.

\section{INTRODUCTION}

Volcanic eruptions are efficient sources of atmospheric pressure perturbations within the infrasound band $(<20 \mathrm{~Hz})$. Volcanic infrasound is a direct measurement of fluctuation at the magma-air interface, and the atmosphere in the vicinity of the vent has a relatively simple Green's function compared to the solid medium through which seismic waves propagate. Hence, volcanic infrasound can carry valuable information about source dynamics without significant loss of source features compared to the seismic counterpart (Vergniolle et al. 1996; Garces \& McNutt 1997; Ozerov et al. 2003; Johnson \& Lees 2010).

A proper acoustic source model is necessary to extract the underlying physics of volcanic eruptions from infrasound observations. Real acoustic sources producing volcanic infrasound have finite dimensions such as radius of bubble or vent. In this case, source geometry and frequency-dependent diffraction must be considered (Kim $\&$ Lees 2011). However, if the source region is compact with respect to the wavelength, then the source can be dealt with as a point with magnitude but no spatial dimension. This point source approximation has been widely used in volcanic infrasound studies to quantify acoustic energy produced during volcanic eruptions (Johnson et al. 2004; Johnson 2007; Ripepe et al. 2007; Vergniolle et al. 2004).

A monopole is the simplest and most efficient point source of volcanic infrasound. Sound generated by a monopole radiates uniformly into all directions. Isotropic mass outflux during volcanic eruptions can be modelled as a monopole source, and volume or velocity of ejecting material can be estimated from acoustic pressure (Woulff \& McGetchin 1976; Johnson et al. 2004).

Forces generated during volcanic activity can be simulated by a dipole source, which has two successive monopoles out of phase by $180^{\circ}$. Pressure disturbance by a dipole source exhibits directivity in the radiation pattern, which depends on the angle between the direction of the dipole and station location. Caplan-Auerbach et al. (2010) used acoustic power observed at Augustine volcano, Alaska, to estimate the velocity of material exiting the vent based on the dipole model.

In both cases, selecting a proper source model is critical for estimating energy flux in the vicinity of a volcanic vent. Most studies of volcanic infrasound have used either a monopole or dipole source exclusively to model acoustic sources of volcanic eruptions, but theoretically both types of acoustic sources can be excited simultaneously. For instance, an unbaffled loudspeaker is theoretically an ideal dipole source. However, sound measurements from the loudspeaker are not explained solely by the dipole model, and the substantial monopole component is also required (Li et al. 1997). Hence, 'multipole' sources can be appropriate for acoustic sources of volcanic infrasound.

In this paper we review the solutions to the scalar wave equation for monopole and dipole sources in a half-space, and then present an inversion method based on the half-space model to estimate source parameters for a monopole and dipole simultaneously. The 
inversion method is applied to infrasound observations from Tungurahua volcano, Ecuador, in 2010 which exhibited remarkable directivity in acoustic radiation. The inversion process successfully estimated stable and consistent source parameters corresponding to the recorded radiation patterns. Consequently, the infrasound radiation pattern observed at Tungurahua volcano is well explained by the multipole source. The dipole component contributes to the observed directivity, and the monopole does to the overall pressure level. The multipole source model in a half-space gives us a powerful method to cope with complicated sources for volcanic infrasound and to understand underlying source physics of volcanic eruptions.

\section{MONOPOLE AND DIPOLE SOURCE MODELS IN A FREE SPACE}

Acoustic radiation fields from sources of limited spatial extent can be described in terms of a multipole series. If the source is acoustically compact, so that its largest dimension is much shorter than a wavelength, the multipole series converges rapidly and the first few terms remain non-zero. Consequently, compact sources are typically approximated as monopole, dipole and quadrupole terms (Rossing 2007) although only monopole and dipole cases are considered in this paper. It is convenient to write the total sound field in terms of a Green's function. Assuming a point source placed at $\boldsymbol{r}_{0}=\left(x_{0}, y_{0}, z_{0}\right)$ in an unbounded atmosphere, the total sound field $p(\boldsymbol{r}, t)$ satisfies the inhomogeneous wave equation

$\nabla^{2} p-\frac{1}{c^{2}} \frac{\partial^{2} p}{\partial t^{2}}=-\delta(t) \delta\left(\boldsymbol{r}-\boldsymbol{r}_{0}\right)$

where $p$ is pressure, $c$ is the sound speed and $r=(x, y, z)$ is a receiver position. If the source is simple-harmonic, so that $\delta(t)$ from the origin can be substituted by $\mathrm{e}^{-\mathrm{i} \omega t}$ the resulting wave motion is $p=\hat{p}(\omega) \mathrm{e}^{-\mathrm{i} \omega t}$, which satisfies the inhomogeneous Helmholtz equation

$\nabla^{2} \hat{p}(\omega)+k^{2} \hat{p}(\omega)=-\delta\left(\boldsymbol{r}-\boldsymbol{r}_{\mathbf{0}}\right)$.

The Green's function solution to the Helmholtz equation (Morse \& Ingard 1986) is

$G_{\omega}\left(\boldsymbol{r} \mid \boldsymbol{r}_{0}\right)=\frac{1}{4 \pi R} \mathrm{e}^{\mathrm{i} k R}$,

where $R=\left|\boldsymbol{r}-\boldsymbol{r}_{0}\right|$. Because this is a solution only for a single frequency, the transient solution in the time domain must be obtained from integration with respect to all frequencies, as follows:

$$
\begin{aligned}
G\left(\boldsymbol{r}, t \mid \boldsymbol{r}_{0}, t_{0}=0\right) & =\int_{-\infty}^{\infty} G_{\omega}\left(\boldsymbol{r} \mid \boldsymbol{r}_{0}\right) \mathrm{e}^{-\mathrm{i} \omega t} \mathrm{~d} \omega \\
& =\int_{-\infty}^{\infty} \frac{1}{4 \pi R} \mathrm{e}^{-\mathrm{i} \omega(t-R / c)} \mathrm{d} \omega \\
& =\frac{\delta(t-R / c)}{4 \pi R} .
\end{aligned}
$$

$G\left(\boldsymbol{r}, t \mid \boldsymbol{r}_{\mathbf{0}}, t_{0}\right)$ is the Green's function for eq. (1), and $G_{\omega}\left(\boldsymbol{r} \mid \boldsymbol{r}_{\mathbf{0}}\right)$ can be interpreted as the Fourier transform pair of $G\left(\boldsymbol{r}, t \mid \boldsymbol{r}_{0}, t_{0}\right)$. Expressions for monopole and dipole sources can be derived subsequently from the Green's function.

\subsection{Monopole source}

A point monopole is the simplest source for sound (Fig. 1a). Consider the radiation from a pulsating sphere with a small radius. If the mass flow of fluid from the source is $S(t)$, which is called the monopole strength hereafter, the excess pressure radiated from a monopole can be expressed as (Morse \& Ingard 1986)

$\nabla^{2} p-\frac{1}{c^{2}} \frac{\partial^{2} p}{\partial t^{2}}=-\dot{S}(t) \delta\left(\boldsymbol{r}-\boldsymbol{r}_{\mathbf{0}}\right)$,

where $\dot{S}(t)$ is the mass flow rate per unit time, or mass acceleration in units of $\rho \mathrm{m}^{3} \mathrm{~s}^{-2}$ where $\rho$ is the fluid density in $\mathrm{kg} \mathrm{m}^{-3}$. If the source is harmonic such as $S(t)=\hat{S}(\omega) \mathrm{e}^{-\mathrm{i} \omega t}$, the solution can be expressed in terms of the Green's function (eq. 3)

$$
\begin{aligned}
\hat{p}(\omega) & =-\mathrm{i} k c \hat{S}(\omega) G_{\omega} \\
& =-\frac{\mathrm{i} k c \hat{S}(\omega)}{4 \pi R} \mathrm{e}^{\mathrm{i} k R} .
\end{aligned}
$$

The transient solution becomes

$$
\begin{aligned}
p(\boldsymbol{r}, t) & =\int_{-\infty}^{\infty} \hat{p}(\omega) \mathrm{e}^{-\mathrm{i} \omega t} \mathrm{~d} \omega \\
& =\frac{1}{4 \pi R} \dot{S}\left(t-\frac{R}{c}\right) .
\end{aligned}
$$

The pressure at any point $\boldsymbol{r}$ in the field is omni-directional and determined by the mass acceleration $\dot{S}(t)$ of the simple source. A body of oscillating volume, such as a boxed loudspeaker at low frequencies, is a good example of a monopole source.

\subsection{Dipole source}

To generate the sound field from a point monopole, fluid must be introduced or withdrawn from a small region of space. In the dipole case sound can be produced by moving a portion of the fluid back and forth with no net introduction of fluid. The dipole source is simulated by a neighbouring pair of equal point monopoles with opposite signs. In this way fluid is being 'breathed in' by one source as it is being 'breathed out' by the other source. If the source of strength $S(t)$ is at $\boldsymbol{r}_{0}+\frac{1}{2} \boldsymbol{d}$ and the one of strength $-S(t)$ is at $\boldsymbol{r}_{0}-\frac{1}{2} \boldsymbol{d}$, and the vector distance $(\boldsymbol{d})$ between the two sources (Fig. 1b) is very small compared with the wavelength, then the wave equation for the dipole source can be expressed as (Morse \& Ingard 1986)

$$
\begin{aligned}
\nabla^{2} p-\frac{1}{c^{2}} \frac{\partial^{2} p}{\partial t^{2}} & =\nabla \cdot\left[\dot{S}(t) \boldsymbol{d} \delta\left(\boldsymbol{r}-\boldsymbol{r}_{\mathbf{0}}\right)\right] \\
& =-\dot{\boldsymbol{D}}(t) \cdot \nabla_{0} \delta\left(\boldsymbol{r}-\boldsymbol{r}_{\mathbf{0}}\right) .
\end{aligned}
$$

$\boldsymbol{D}(t) \equiv S(t) \boldsymbol{d}$ is momentum in units of $\mathrm{kg} \cdot \mathrm{m} \mathrm{s}^{-1}$, and the operator $\nabla_{0}$ denotes the gradient with respect to the source coordinates, $\boldsymbol{r}_{\mathbf{0}}$. Provided a harmonic source, $\boldsymbol{D}(t)=\hat{\boldsymbol{D}}(\omega) \mathrm{e}^{-\mathrm{i} \omega t}$,

$$
\begin{aligned}
\hat{p}(\omega)= & -\mathrm{i} k c \hat{\boldsymbol{D}}(\omega) \cdot \nabla_{0} G_{\omega}\left(\boldsymbol{r} \mid \boldsymbol{r}_{0}\right) \\
= & -\frac{k^{2} c}{4 \pi R}\left[1+\frac{\mathrm{i}}{k R}\right] \mathrm{e}^{\mathrm{i} k R} \\
& \times\left[\frac{\left(x-x_{0}\right)}{R} \hat{D}_{x}+\frac{\left(y-y_{0}\right)}{R} \hat{D}_{y}+\frac{\left(z-z_{0}\right)}{R} \hat{D}_{z}\right] .
\end{aligned}
$$

In the far field where the distance is much longer than the wavelength, the condition, $k R \gg 1$, is satisfied. Furthermore, if $\boldsymbol{r}_{\mathbf{0}}=0$ so that $|\boldsymbol{r}|=R=r$, eq. (9) can be approximated as

$$
\begin{aligned}
\hat{p}(\omega) & \simeq-\frac{k^{2} c}{4 \pi r}\left[\frac{x}{r} \hat{D}_{x}+\frac{y}{r} \hat{D}_{y}+\frac{z}{r} \hat{D}_{z}\right] \mathrm{e}^{\mathrm{i} k R} \\
& =-\frac{k^{2} c}{4 \pi r}\left|\boldsymbol{D}_{\omega}\right| \cos \theta \mathrm{e}^{\mathrm{i} k R},
\end{aligned}
$$

where $\theta$ is the angle between the dipole vector and the $z$ coordinate axis (Fig. 1b). The sound field generated by the dipole shows directivity which depends on the angle $\theta$. The magnitude of the pressure 

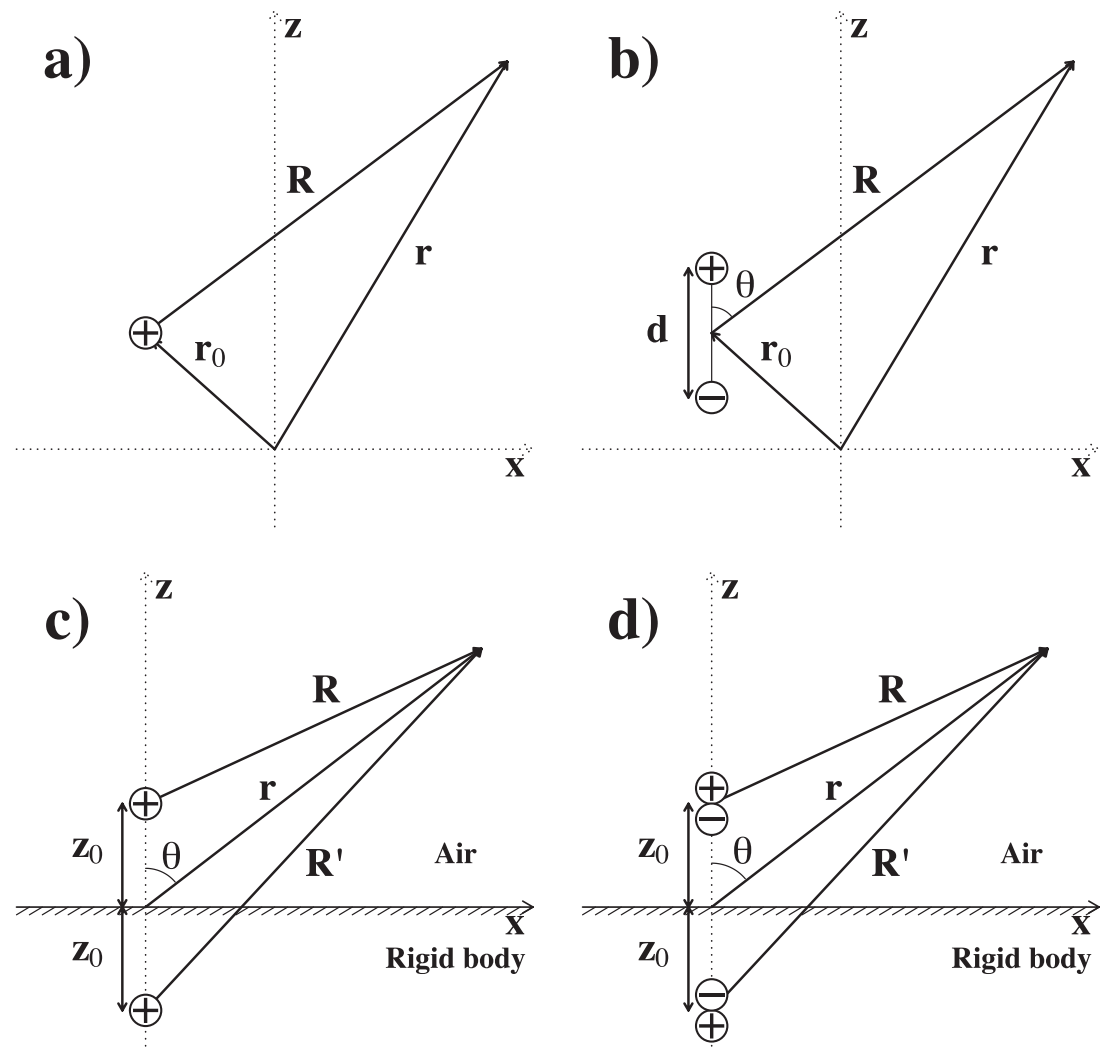

Figure 1. Geometric configurations for (a) monopole in a free space, where $\mathbf{r}_{\mathbf{0}}$ and $\mathbf{r}$ are source and receiver positions, and $\mathbf{R}$ is the distance between the source and the receiver. (b) Dipole in a free space, where $\mathbf{d}$ is the distance between two opposite point sources. (c) Monopole in a half-space, where $\mathbf{z}_{0}$ is the distance between a source and a half-space, and $\mathbf{R}^{\prime}$ is the distance from the image of the source to a receiver. (d) Dipole in a half-space

disturbances is maximum on the dipole axis, and zero at $90^{\circ}$. This dipole solution can be separated into two components: the vertical and horizontal. Any arbitrary dipole can be decomposed into vertical and horizontal dipoles and the sound field can be rewritten, as follows:

$\hat{p}_{H}(\omega)=-\frac{k^{2} c}{4 \pi r}\left[\frac{x}{r} \hat{D}_{x}+\frac{y}{r} \hat{D}_{y}\right] \mathrm{e}^{\mathrm{i} k R}$,

$\hat{p}_{V}(\omega)=-\frac{k^{2} c}{4 \pi r}\left[\frac{z}{r} \hat{D}_{z}\right] \mathrm{e}^{\mathrm{i} k R}$,

where $\hat{p}_{H}(\omega)$ and $\hat{p}_{V}(\omega)$ are the sound fields generated by the horizontal and vertical dipole, respectively. By considering the vertical and horizontal dipole components of the total sound field independently, it is easier to establish the effects of a solid boundary described, in the following section.

\section{MONOPOLE AND DIPOLE SOURCE MODELS IN A HALF-SPACE}

In the far field, volcanic infrasound can be considered to propagate in a half-space. In this range and at low frequencies, irregular topography can be ignored so that the solid boundary can be approximated as being flat. Even though the ground surface absorbs some acoustic energy, especially in the high-frequency range, it is assumed to be a rigid boundary due to a high contrast in acoustic velocity. If the plane is perfectly rigid, then the boundary condition requires that the normal fluid velocity is zero at the surface. This boundary condition can most easily be met using the image method.
An image, $S^{\prime}$, having the same phase and magnitude as the source, $S$, is placed a distance $z_{0}$ below the boundary, and the boundary is removed. In other words the rigid part is replaced by air (Fig. 1c). The resulting sound waves generated by both source and image radiate into unbounded space satisfying the boundary condition. Naturally, only the region above the boundary plane contains the medium and carries acoustic energy. Conversely, the region below the boundary has no physical reality. Mathematically, the wavefield reflecting from the rigid plane represents the superposition of two wavefields generated by both source and image. Hence, the Green's function for a half-space can be written in terms of two Green's functions in a free space (Morse \& Ingard 1986)

$g_{\omega}\left(\boldsymbol{r} \mid \boldsymbol{r}_{\mathbf{0}}\right)=G_{\omega}\left(\boldsymbol{r} \mid \boldsymbol{r}_{\mathbf{0}}\right)+G_{\omega}\left(\boldsymbol{r} \mid \boldsymbol{r}_{\mathbf{0}}^{\prime}\right)$,

where $\boldsymbol{r}_{\mathbf{0}}=\left(x_{0}, y_{0}, z_{0}\right), \boldsymbol{r}_{\mathbf{0}}^{\prime}=\left(x_{0}, y_{0},-z_{0}\right)$, and $G_{\omega}\left(\boldsymbol{r} \mid \boldsymbol{r}_{\mathbf{0}}^{\prime}\right)$ is Green's function for the image in a free space.

\subsection{Monopole source}

Monopole radiation in a half-space can be easily solved by replacing the Green's function, $G_{\omega}$ in eq. (6) with $g_{\omega}$

$$
\begin{aligned}
\hat{p}(\omega) & =-\mathrm{i} k c \hat{S}(\omega) g_{\omega}\left(\boldsymbol{r} \mid \boldsymbol{r}_{\mathbf{0}}\right) \\
& =-\mathrm{i} k c \hat{S}(\omega)\left[G_{\omega}\left(\boldsymbol{r} \mid \boldsymbol{r}_{\mathbf{0}}\right)+G_{\omega}\left(\boldsymbol{r} \mid \boldsymbol{r}_{\mathbf{0}}^{\prime}\right)\right] \\
& =-\mathrm{i} k c \hat{S}(\omega)\left[\frac{1}{4 \pi R} \mathrm{e}^{\mathrm{i} k R}+\frac{1}{4 \pi R^{\prime}} \mathrm{e}^{\mathrm{i} k R^{\prime}}\right] .
\end{aligned}
$$

At distances $R$ from the source, which are much larger than the source is from the origin (i.e. for $R \gg r_{0}$ ), the two waves combine 

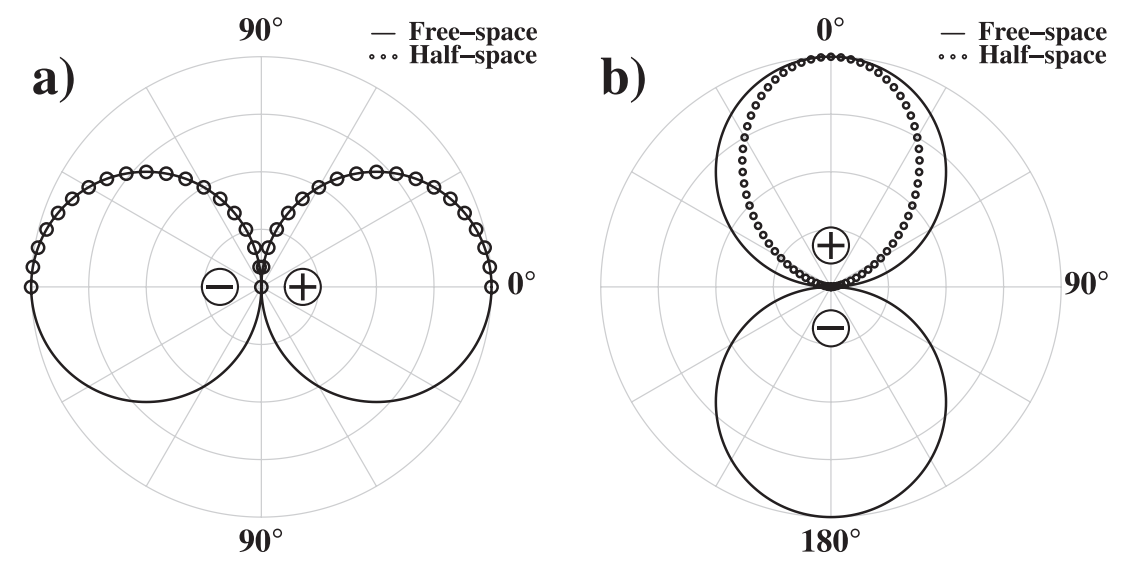

Figure 2. Theoretical directivity patterns for far-field sound pressure radiation from (a) horizontal dipole (eqs 12 and 21 ) and (b) vertical dipole (eqs 13 and 23). Pressures are normalized to their own maximum amplitudes.

to form what appears to be a single, non-simple source at the origin. When $r \gg r_{0}$ and $\boldsymbol{r}_{0}=\left(0,0, z_{0}\right)$,

$R=\left|\boldsymbol{r}-\boldsymbol{r}_{0}\right| \simeq r-z_{0} \cos \theta$,

$R^{\prime} \simeq r+z_{0} \cos \theta$

then,

$$
\begin{aligned}
\hat{p}(\omega) & \simeq-\frac{\mathrm{i} k c \hat{S}(\omega)}{2 \pi r} \cos \left(k z_{0} \cos \theta\right) \mathrm{e}^{\mathrm{i} k r} \\
& \simeq-\frac{\mathrm{i} k c \hat{S}(\omega)}{2 \pi r} \mathrm{e}^{\mathrm{i} k r} \quad\left(\text { if } k z_{0} \ll 1\right) .
\end{aligned}
$$

If $k z_{0} \ll 1$, that is, if the location of the source above the boundary is considerably less than a wavelength, the far field is very weakly dependent on $\theta$, thus resembling the far field from a simple source with strength $2 \hat{S}(\omega)$ at the origin. The effective strength is doubled because the reflected wave adds to the initial wave in this case; the source and image are close enough together to be considered a single source of double strength. The transient solution (Lighthill 1978) in the time domain becomes

$p(\boldsymbol{r}, t)=\frac{1}{2 \pi r} \dot{S}\left(t-\frac{r}{c}\right)$.

\subsection{Dipole source}

As in the monopole case, dipole radiation in a half-space can be obtained from the Green's function shown in eq. (14). If the source at $z_{0}$ is a dipole of strength $\hat{\boldsymbol{D}}(\omega)$ inclined at angle $\theta$ with respect to the vertical axis, then the mirror image will have the same $x$ and $y$ components as the source, but a $D_{z}$ opposite in sign from that of the source. Therefore the total sound field is

$$
\begin{aligned}
\hat{p}(\omega) & =-\mathrm{i} k c \hat{\boldsymbol{D}}(\omega) \cdot \nabla_{0} g_{\omega}\left(\boldsymbol{r} \mid \boldsymbol{r}_{\mathbf{0}}\right) \\
& =-\mathrm{i} k c \hat{\boldsymbol{D}}(\omega) \cdot \nabla_{0}\left[G_{\omega}\left(\boldsymbol{r} \mid \boldsymbol{r}_{\mathbf{0}}\right)+G_{\omega}\left(\boldsymbol{r} \mid \boldsymbol{r}_{\mathbf{0}}^{\prime}\right)\right] .
\end{aligned}
$$

Using the same approximations for $R$ and $R^{\prime}$, and $k r \gg 1$ the far-field solution can be obtained as (Morse \& Ingard 1986)

$$
\begin{aligned}
\hat{p}(\omega) \simeq-\frac{k^{2} c}{2 \pi r}[ & \left(\frac{x}{r} \hat{D}_{x}+\frac{y}{r} \hat{D}_{y}\right) \cos \left(k z_{0} \cos \theta\right) \\
& \left.-\mathrm{i} \frac{z}{r} \hat{D}_{z} \sin \left(k z_{0} \cos \theta\right)\right] \mathrm{e}^{\mathrm{i} k r} \\
=- & \frac{k^{2} c}{2 \pi r}\left[\left(\frac{x}{r} \hat{D}_{x}+\frac{y}{r} \hat{D}_{y}\right)-\mathrm{i} \frac{z}{r} \hat{D}_{z} k z_{0} \cos \theta\right] \mathrm{e}^{\mathrm{i} k r} .
\end{aligned}
$$

It is convenient to split the sound fields into two parts corresponding to the horizontal $\left(\hat{p}_{H}\right)$ and vertical dipole $\left(\hat{p}_{V}\right)$ sources

$$
\begin{aligned}
\hat{p}_{H}(\omega) & =-\frac{k^{2} c}{2 \pi r}\left(\frac{x}{r} \hat{D}_{x}+\frac{y}{r} \hat{D}_{y}\right) \mathrm{e}^{\mathrm{i} k r} \\
= & -\frac{k^{2} c}{2 \pi r} \hat{D}_{H} \cos \phi \mathrm{e}^{\mathrm{i} k R} \\
\hat{p}_{V}(\omega) & =\frac{\mathrm{i} k^{3} c}{2 \pi r}\left(\frac{z}{r} \hat{Q}_{z z} \cos \theta\right) \mathrm{e}^{\mathrm{i} k r} \\
= & \frac{\mathrm{i} k^{3} c}{2 \pi r} \hat{Q}_{z z} \cos ^{2} \theta \mathrm{e}^{\mathrm{i} k r},
\end{aligned}
$$

where $\hat{D}_{H}=\sqrt{\hat{D}_{x}^{2}+\hat{D}_{y}^{2}}, \phi$ is an azimuth for the horizontal dipole axis and $\hat{Q}_{z z}=\hat{D}_{z} z_{0}$. Since the dipole and its image become superimposed at large distances and for long wavelengths, the effective strength of the horizontal dipole above the half-space is doubled. The directivity pattern still depends on $\cos \phi$ as does the sound field from the horizontal dipole in a free space (Fig. 2a). The radiation pattern from the vertical dipole is, however, different from that of a free space. The effective strength is not just twice that of the source in a free space, but depends on the distance $z_{0}$ from the boundary. The radiation pattern for the vertical dipole shows the $\cos ^{2} \theta$ directivity. The pressure disturbances have a maximum magnitude on the dipole axis and attenuate much faster than that of the vertical dipole in a free space as the angle $\theta$ approaches to $90^{\circ}$ (Fig. 2b). This radiation pattern is that of a longitudinal quadrupole in a free space, as one would expect from Fig. 1(d) (Pierce 1989).

The transient solutions for the far field are obtained as follows:

$$
\begin{aligned}
p_{H}(\boldsymbol{r}, t) & =\int_{-\infty}^{\infty} \hat{p}_{H}(\omega) \mathrm{e}^{-\mathrm{i} \omega t} \mathrm{~d} \omega \\
& =\frac{1}{2 \pi r c}\left[\frac{x}{r} \ddot{D}_{x}(t-r / c)+\frac{y}{r} \ddot{D}_{y}(t-r / c)\right], \\
p_{V}(\boldsymbol{r}, t) & =\int_{-\infty}^{\infty} \hat{p}_{V}(\omega) \mathrm{e}^{-\mathrm{i} \omega t} \mathrm{~d} \omega \\
& =\frac{1}{2 \pi r c^{2}}\left[\frac{z}{r} \frac{\partial^{3} Q_{z z}\left(t-\frac{r}{c}\right)}{\partial t^{3}} \cos \theta\right] .
\end{aligned}
$$

These solutions can be used for the inversion of the source time function using the method described in Section 4. 


\section{INVERSION FOR SOURCE PARAMETERS}

The inversion method for acoustic source parameters was developed based on the half-space model. An acoustic source from a volcano might be simulated as a combination of a monopole, dipole and quadrupole in the view of the point source approximation. In this paper only monopole and dipole sources are taken into account. Since the source dimension is small with respect to the wavelength, quadrupole sources are comparatively less efficient. By excluding quadrupole sources the inverse problem is simplified. Even though quadrupole sources are not included in our inversion scheme, volcanic infrasound with large-scale jet noise may be affected by quadrupole sources (Matoza et al. 2009). A combined monopole/dipole source radiating into a half-space can be expressed as follows:

$p(\boldsymbol{x}, t)=p_{M}+p_{H}+p_{V}$,

where $p_{M}, p_{H}$ and $p_{V}$ are the sound field excited by a monopole, horizontal dipole and vertical dipole, respectively. In many field experiments, acoustic sensors are placed only on the ground which can be considered as a horizontal plane. Because the acoustic pressure from the vertical dipole decreases steeply as its deviation from the dipole axis increases (Fig. 2b and eq. 24), $p_{V}$ produces only a small contribution to the total sound field near the ground surface. Inversely, low level noise with observations recorded near the ground can induce large errors in source estimates for the vertical dipole. By ignoring the vertical dipole, pressure disturbances near the surface are rewritten in terms of eqs (18) and (25):

$p(\boldsymbol{r}, t)=\frac{1}{2 \pi r}\left[\dot{S}\left(t-\frac{r}{c}\right)+\frac{x}{c r} \dot{F}_{x}\left(t-\frac{r}{c}\right)+\frac{y}{c r} \dot{F}_{y}\left(t-\frac{r}{c}\right)\right]$.

The horizontal dipole was defined as $\boldsymbol{F} \equiv \dot{\boldsymbol{D}}$, which is force in units of $\mathrm{kg} \cdot \mathrm{m} \mathrm{s}^{-2}$. A set of linear equations can be derived from approximation of a continuous relationship by a discrete representation. Let $p_{i}$ be the infrasound record obtained from the $i$ th station,

$p_{i}^{k} \equiv p_{i}\left(t_{0}+k \Delta t-\frac{r}{c}\right)$

so that $p_{i}^{k}$ is the $k$ th element of the time-series. The model vector which contains unknown parameters is defined as follows:

$$
\begin{aligned}
\boldsymbol{m}^{k} & =\left[m_{1}^{k}, m_{2}^{k}, m_{3}^{k}\right] \\
& =\left[\dot{S}\left(t_{0}+k \Delta t\right), \dot{F}_{x}\left(t_{0}+k \Delta t\right), \dot{F}_{y}\left(t_{0}+k \Delta t\right)\right] .
\end{aligned}
$$

From eq. (28), the relationship between the observed data and the model vector is obtained,

$p_{i}^{k}=\frac{1}{2 \pi r_{i}}\left[m_{1}^{k}+\frac{x_{i}}{c r_{i}} m_{2}^{k}+\frac{y_{i}}{c r_{i}} m_{3}^{k}\right]$.

These linear equations allow a direct inversion of the infrasound records to obtain an estimate of the acoustic source parameters, as characterized by monopole strength and dipole vector. In presenting the details of the actual inverse method, it is convenient to write eq. (31) in the common matrix form,

$\boldsymbol{P}^{k}=\boldsymbol{G} \boldsymbol{m}^{k}$.

In this case, $\boldsymbol{P}^{k}$ is a vector of dimension $n$ and is composed of sampled pressure disturbances observed from $n$ stations. The matrix $\boldsymbol{G}$ is an $n \times 3$ matrix. To solve eq. (32), the number of stations must be larger than three. Singular value decomposition of $\boldsymbol{G}$ is used and generalized inverse $\boldsymbol{G}^{-1}$ (Parker 1994) is calculated. $\boldsymbol{G}$ can be decomposed as

$\boldsymbol{G}=\boldsymbol{U} \boldsymbol{S} \boldsymbol{V}^{T}$

where $\boldsymbol{U}$ consists of the eigenvectors associated with the non-zero eigenvalues of $\boldsymbol{G} \boldsymbol{G}^{T}, \boldsymbol{V}$ consists of similar eigenvectors for $\boldsymbol{G}^{T} \boldsymbol{G}$, and the diagonal members of $\boldsymbol{S}$ are the positive square roots of the non-zero eigenvalues of $\boldsymbol{G}^{T} \boldsymbol{G}$. The generalized inverse of $\boldsymbol{G}$ becomes

$\boldsymbol{G}^{-1}=\boldsymbol{V} \boldsymbol{S}^{-1} \boldsymbol{U}^{T}$.

Eq. (32) can be solved by taking the matrix inverse to obtain

$\boldsymbol{m}^{k}=\boldsymbol{G}^{-1} \boldsymbol{P}^{k}$.

This equation provides a very general means of solving the inverse problem but we need to evaluate the significance of the inverse.

\section{STABILITY OF INVERSION METHOD}

To obtain some measure of the fit resulting from the inversion procedure and to quantify the significance of the inversion, the variances of the model parameters are calculated. For statistically independent data the variance of the model becomes (Jackson 1972)

$\operatorname{var}\left(m_{j}^{k}\right)=\sum_{i=1}^{n}\left(G_{j i}^{-1}\right)^{2} \operatorname{var}\left(P_{i}^{k}\right)$.

As shown in eq. (34), $\left(G_{k j}^{-1}\right)^{2}$ is proportional to the reciprocal of the eigenvalues of $\boldsymbol{G}^{T} \boldsymbol{G}$. Small eigenvalues will therefore lead to high uncertainty in $m^{k}$ terms lowering the stability of the inversion. The stability can be characterized by the condition number of the problem (Stump \& Johnson 1977), defined as the ratio of the largest to smallest eigenvalues of $\boldsymbol{G}^{\boldsymbol{T}} \boldsymbol{G}$. Since the matrix $\boldsymbol{G}$ depends on receiver position and the sound speed $\left(\simeq 340 \mathrm{~m} \mathrm{~s}^{-1}\right)$ in air according to eq. (32), azimuthal distribution of stations is critical to the condition number.

A set of experiments examined the azimuthal dependency of the condition number. Source time functions for a monopole and dipole are taken to be Gaussian functions with different wavelengths. From these sources, synthetic data were generated with 10 per cent Gaussian random noise for each station. Six different distributions of stations were tested and the condition number, model parameters and their variances were calculated using eqs (35) and (36). Mass flux $S(t)$ and the dipole vector $\boldsymbol{F}(t)$ were integrated from the model parameters (eq. 30). The dipole vector was denoted by the magnitude $|\boldsymbol{F}|$ and the azimuth $\theta$. The error associated with the numerical integration was ignored. Typically, for the trapezoid rule, the error terms are on the order of the square of time interval $\left(\Delta t^{2}\right)$ of the data (Cheney \& Kincaid 2007). The exact magnitude of the error cannot be calculated without the original analytic function. Each configuration and results of each trial are given in Table 1 .

While the inversion was successful, rather large condition numbers were yielded in Trials 1 and 2. Trials 3 and 4 also show successful inversion, although their condition numbers and standard deviations are significantly reduced. The result of fitting the data and estimated source parameters from Trials 1 and 4 are compared in Fig. 3. Even though both trials show good fits to the synthetic data, only the estimated source parameters from Trial 4 are reliable. Therefore, to achieve stable inversion, at least three stations covering $180^{\circ}$ of azimuth are required. In Trials 5 and 6 , the condition numbers are not reduced compared to Trial 4, though the standard 
Table 1. Estimates of the source parameters and their standard deviations for different station configurations.

\begin{tabular}{cccccc}
\hline Trial no. & Station azimuth $\left(^{\circ}\right)$ & Condition no. & $S$ & $|F|$ & $\theta$ \\
\hline Source & & & $10 \mathrm{~kg} \mathrm{~s}^{-1}$ & $3000 \mathrm{~kg} \mathrm{~m} \mathrm{~s}^{-2}$ & $70^{\circ}$ \\
1 & $0,15,30$ & $4.4 \times 10^{8}$ & $0.4( \pm 29)$ & $2817( \pm 8677)$ & $150^{\circ}( \pm 112)$ \\
2 & $0,45,90$ & $6.0 \times 10^{6}$ & $11( \pm 2.9)$ & $2755( \pm 887)$ & $73^{\circ}( \pm 19)$ \\
3 & $0,90,180$ & $5.2 \times 10^{5}$ & $10( \pm 0.61)$ & $2741( \pm 344)$ & $67^{\circ}( \pm 5)$ \\
4 & $0,120,240$ & $2.3 \times 10^{5}$ & $9.9( \pm 0.54)$ & $2822( \pm 271)$ & $71^{\circ}( \pm 5)$ \\
5 & $0,90,180,270$ & $2.3 \times 10^{5}$ & $10( \pm 0.46)$ & $3080( \pm 234)$ & $67^{\circ}( \pm 4)$ \\
6 & $0,45,90,135,180,225,270,315$ & $2.3 \times 10^{5}$ & $9.8( \pm 0.33)$ & $3059( \pm 163)$ & $70^{\circ}( \pm 2.9)$ \\
\hline
\end{tabular}

deviations decrease continuously. The experiment suggests that doing the inversion at least three stations evenly distributed over $360^{\circ}$ should produce the best result for the inversion.

\section{INFRASOUND RADIATION PATTERN AND SOURCE CHARACTERISTICS AT TUNGURAHUA VOLCANO}

\subsection{Field experiment}

Tungurahua volcano is a large andesitic stratovolcano in the Cordillera Real of Ecuador. The active vent, $5023 \mathrm{~m}$ high, is located on the upper part of its northwestern flank. In 2010 May, a new eruptive cycle began with a mid-size volcanic explosions associated with sustained ash column emissions, pyroclastic flows and seismic and infrasonic tremor.

Between 2006 and 2010 a network of five broad-band seismoacoustic stations was deployed by IGEPN (Instituto GeofísicoEscuela Politécnica Nacional, Ecuador), with support from Japan's JICA program to monitor Tungurahua for hazard mitigation and volcano research. Each station included an ACO Type-7144/4144 acoustic sensor. The nominal infrasound sensor response was 0.1-100 Hz, with microphone sensitivity $0.025 \mathrm{~V} \mathrm{~Pa}^{-1}$ and output voltage $\pm 5 \mathrm{~V}$. The amplifier sensors were set to record $893.5 \mathrm{~Pa}$ at full scale with sensitivity $-0.005593 \mathrm{~V} \mathrm{~Pa}^{-1}$, and a $100 \mathrm{~Hz}$ lowpass filter was applied in the amplifier circuits. The microphones were designed specifically to record in harsh volcanic settings. Distances between the vent and stations range from $5.05 \mathrm{~km}$ at BPAT (Fig. 4) to $6.11 \mathrm{~km}$ at the furthest station BRUN.

Numerous infrasonic events were recorded during the period of May 28-June 5. Tungurahua infrasound records are characterized by short impulsive onsets indicating explosive eruptions. The peak magnitudes of these events were very large, up to hundreds of Pascals. The infrasound field recorded on the network exhibited clear directivity concerning radiation patterns: this strong directivity is not common in volcano infrasound. In most cases, the highest amplitudes were observed at station BBIL (Fig. 4). Although BPAT is the closest station from the active vent, recorded peak amplitudes were less than those at BBIL. The directivity of the radiation patterns cannot be explained by a simple monopole source. Accordingly, a model with a dipole source may be required.

\subsection{Inversion for source parameters}

The multipole source model (monopole and dipole) was applied to the infrasound records from Tungurahua, and the waveforms were inverted for source parameters. Several assumptions were made: (1) We assumed that infrasound waves from Tungurahua propagate into a half-space. Because of the slope $\left(\approx 20^{\circ}\right)$ of Tungurahua, infrasound spreads out over wider region than that of a hemispherical half-space. In this case, inferred source strength from the half-space assumption is less than that of the 'true' source. (2) The acoustic wave intrinsic attenuation was ignored. Within the lower atmosphere, the attenuation coefficient for frequencies ranging from 0.05 to $4 \mathrm{~Hz}$ is smaller than about $10^{-6} \mathrm{~dB} \mathrm{~m}^{-1}$, which corresponds to a $0.1 \mathrm{~dB}$ loss over a $100 \mathrm{~km}$ path length (Sutherland \& Bass 2004; de Groot-Hedlin 2008). Hence over the 5-10 km distance in our experiment, intrinsic attenuation is negligible. (3) Secondary propagation effects such as reflection, refraction and diffraction were not considered. At short range and low elevation, the atmosphere is considered to be homogeneous. Irregular topography was also ignored. BULB and BRUN were potentially affected by reflection or refraction from local complex geometry. However, because there were no barriers in the line between the vent and the stations and only short impulsive events were chosen, the first single oscillation of signal is likely to be less affected by reflection and refraction. (4) Wind effects are also ignored. Wind usually affects the infrasound amplitude: a station in the upwind direction records larger amplitudes than one in the downwind direction. Theoretically if the wind speed is Mach number 1, then the ratio of upwind to downwind amplitude is about 1.5 in a homogeneous atmosphere (Ostashev et al. 2005). During deployments in 2010 May and June, winds had a mean velocity of $6.3 \pm 5.5 \mathrm{~m} \mathrm{~s}^{-1}$ to the WNW direction, estimated from wind models of the Ecuadorean Civil Aviation Agency. With such low speeds, wind effects on the sound amplitude are negligible. Since the peak amplitude ratio of BBIL to BPAT in most cases exceeds 2 or 3, the amplitude difference is likely caused by acoustic source characteristics rather than wind.

We selected 80 impulsive events during the period of the experiment, using only data with high signal-to-noise ratio $(40 \pm 7 \mathrm{~dB})$. The $6 \mathrm{~s}$ length signals were inverted and source time functions for a monopole and dipole were simultaneously estimated using eq. (32). Integrating the source time function, the monopole strength $S(t)$, horizontal dipole strength $F(t)$ and azimuth of the dipole axis were obtained (Fig. 5).

Because the amount of noise associated with the observations is unknown, it is impossible to calculate exact variances of inverted model parameters using eq. (36). On the assumption of 10 per cent noise with respect to maximum signal amplitude, theoretical variances of the model parameters can be estimated (Table 2). These variances do not represent 'true' uncertainties underlying model estimates, but rather provide insight on how stable model parameters with respect to data variability. Since the signal-to-noise ratios for the selected events are high $(\geq 33 \mathrm{~dB})$, potential error magnitudes associated with the events are not expected to exceed 10 per cent of the signal amplitudes.

\subsection{Results}

Two examples of the data fitting and estimated source parameters are provided in Fig. 5. In both cases the inversion results exhibit reasonably good fits to the data. The largest misfits were associated 
a) Trial 1
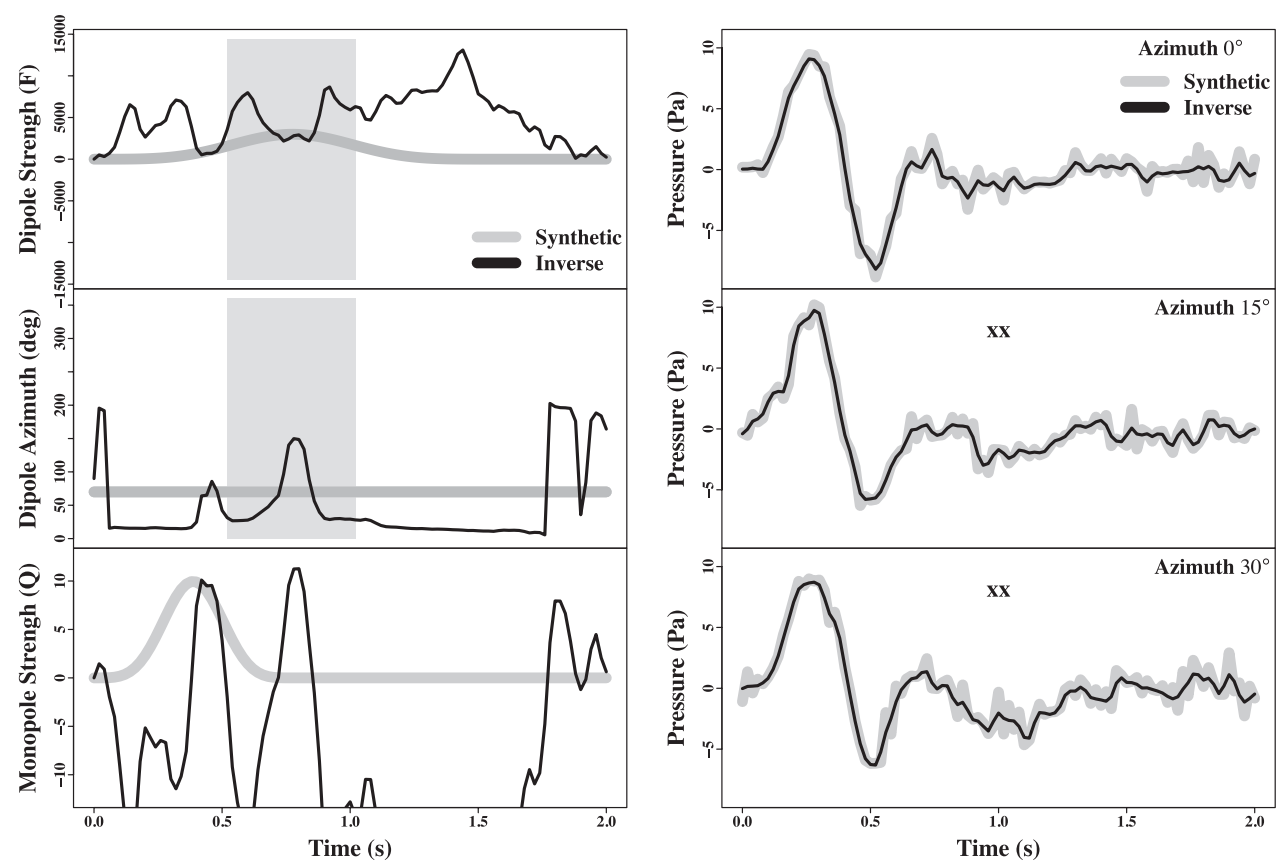

b) Trial 4
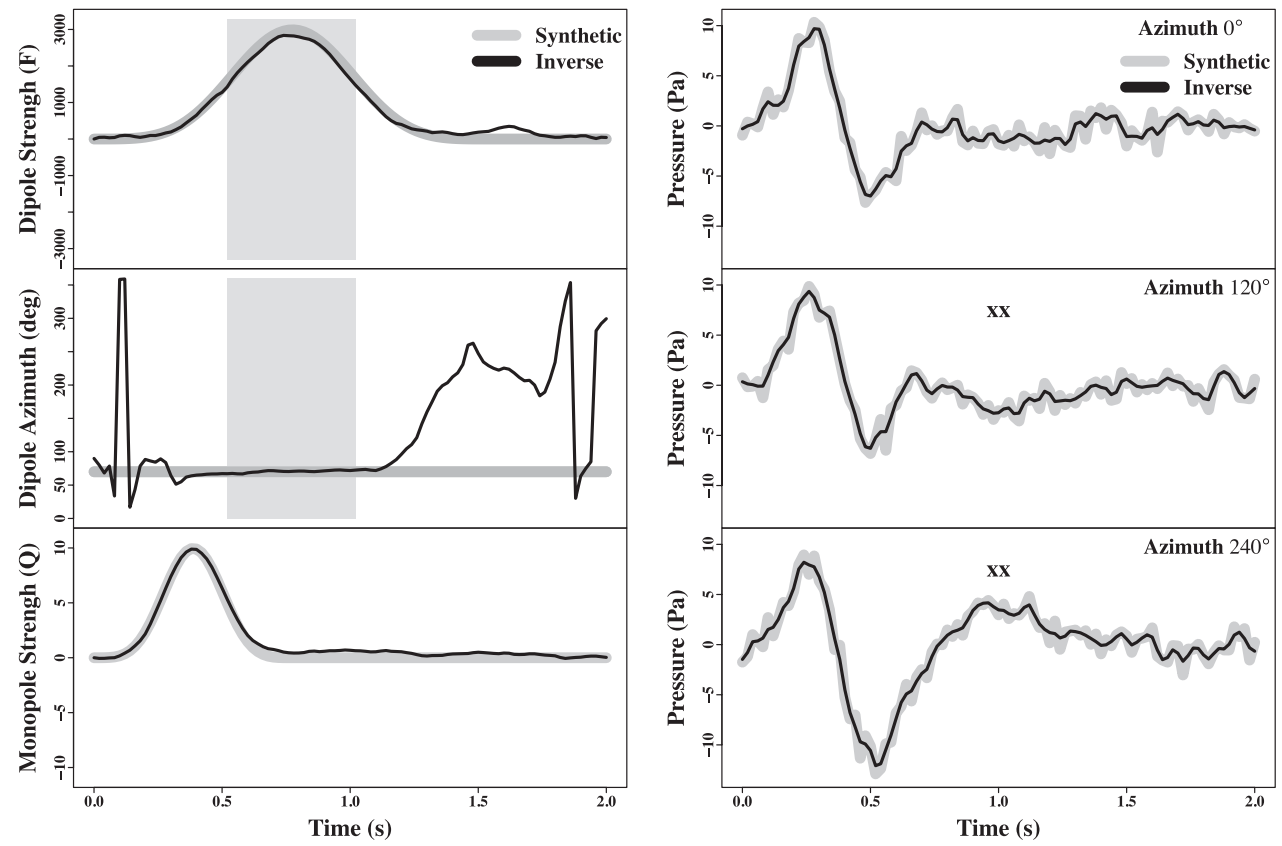

Figure 3. Comparison of Trial 1 (a) and 4 (b): Left columns show estimated source parameters. Fitted infrasound waveforms are given in the right columns. 10 per cent Gaussian random noise is added to the synthetic data. Both trials show good fits to the data. However, only source parameters from Trial 4 are reliable. Estimates of the dipole direction of Trial 4 show good agreements with those of the synthetic dipole in the shaded region where the dipole strength is above noise level.

with stations BRUN and BULB. While the observed amplitudes from BRUN were consistently smaller than the fits, BULB showed larger amplitudes than expected from the inversion. These two stations were located in areas of complex terrain, with two wide and deep valleys nearby. Scattering and reflections may be caused by the complex topography and terrain fluctuation. However, our inversion procedure appears to be stable and the results are consistent (Figs 5 and 6). We surmise that this is because we have used all the available stations for inversion and site effects are not appreciable, leading to predicted pressures that are close to observations.

The condition number for the network configuration is $3.0 \times$ $10^{5}$ (Table 2), larger than $2.3 \times 10^{5}$ for Trial 4, but much smaller than for Trial 3 (Table 1). Standard deviations for the monopole and horizontal dipole strengths are within 10 per cent of their peak 


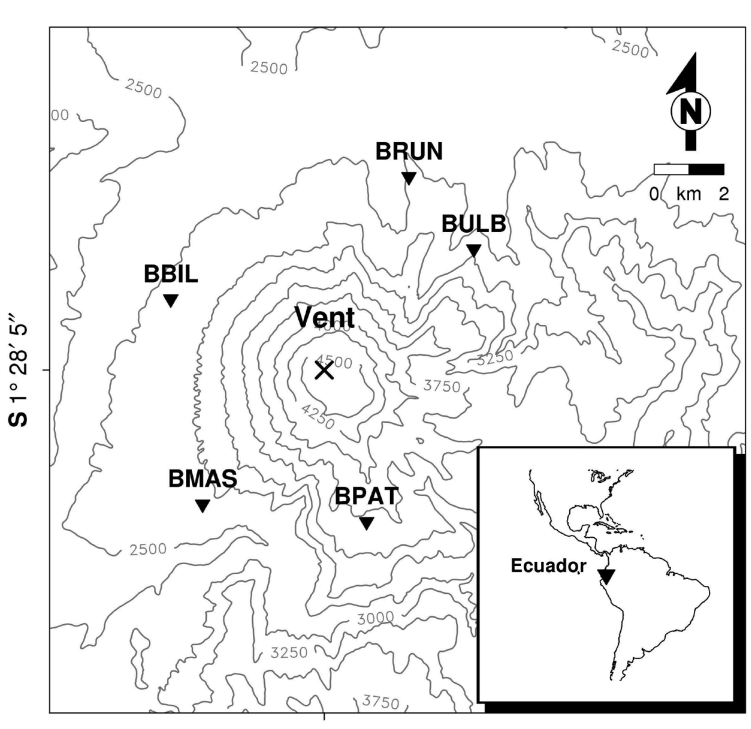

W $78^{\circ} 26^{\prime} 53^{\prime \prime}$

Figure 4. Map of Tungurahua volcano with the station geometry.

amplitudes. The low variances and associated condition numbers suggest that the inversion procedure is stable.

The computed dipole vectors (Fig. 6a) show a consistent direction with mean azimuth $289^{\circ}$. The dipole direction was compared with the crater geometry of Tungurahua. Tungurahua crater has a significant asymmetric shape (Fig. 7). The northwest wall of the crater is $300 \mathrm{~m}$ lower than the southeast wall, and the opening of the crater faces the northwest direction. The asymmetry of this feature was confirmed by visual observation during the field campaign. Taking into account the error of the DEM (produced by Instituto Geofísco Militar with $20 \mathrm{~m}$ resolution) and the geometry changes involved with explosive eruptions, the opening direction and the inferred dipole are considered to align significantly.

We also applied the simultaneous inversion method to 'acoustic noise' that was not related to volcanic eruptions to check the site effects such as an instrument calibration and local noise. Noise data were chosen for different time periods over a week, and the dipole direction was estimated (see the Supporting Information). Dipole directions from the noise were inconsistent with the $289^{\circ}$ azimuth determined from the volcanic source and indicated which stations showed the highest noise level at that time. This suggests that the consistent dipole pattern observed from explosions was not caused by site effects.

Volume flux associated with volcanic explosions can be calculated from the estimated monopole strengths.

$Q=\frac{1}{\rho_{\text {air }}} S(t)$,

where $Q$ is volume flux, $\rho_{\text {air }}=1 \mathrm{~kg} \mathrm{~m}^{-3}$ is the air density, and $S(t)$ is mass flux. The dipole and monopole strengths for events at Tungurahua are well correlated (Fig. 6b). Large explosions presumably generate strong dipole and monopole sources simultaneously. Estimated volume flux ranges between $10^{4}$ and $10^{6} \mathrm{~m}^{3} \mathrm{~s}^{-1}$. Tungurahua volcano has shown a wide range of eruption styles from Strombolian to Vulcanian. During the experiment, it showed Vulcanian explosions with large ash columns and large amount of ballistics (Ruiz et al. 2006; Fee et al. 2010). We compared the volume flux with that of Augustine volcano, Alaska (Caplan-Auerbach et al. 2010). The volume flux of Vulcanian eruptions of Augustine in 2006 were estimated using infrasound observations to range between $2.6 \times$
$10^{5}$ and $6.2 \times 10^{5} \mathrm{~m}^{3} \mathrm{~s}^{-1}$. Because the vent radius of Tungurahua $(\approx 100 \mathrm{~m}$ on $2011 \mathrm{May})$ is larger than that of Augustine $(\approx 30 \mathrm{~m})$, it is reasonable that our estimated volume flux shows wider range, up to the order of $10^{6} \mathrm{~m}^{3} \mathrm{~s}^{-1}$. We note that mass and volume outflux were estimated based on the assumption of the constant standard atmospheric density in the vicinity of the vent. If the vent is overpressurized or the volcanic jet significantly changes the composition of air near the vent, the assumption will likely introduce significant errors in the estimation. Even after taking the error of the density into consideration, however, the volume flux remains comparable to the previous results. This suggests that our multipole analysis is providing reasonable estimates of volume outflux during volcanic explosions.

The magnitude of the dipole vectors was compared with those calculated from observations at Mt Erebus, Antarctica (Johnson et al. 2008). The dipole vector from bubble bursts at the Mt Erebus lava lake was estimated using an acoustic dipole solution in a free space. The resultant dipole strength has a magnitude on the order of $10^{7} \mathrm{~kg} \mathrm{~m} \mathrm{~s}^{-2}$. The acoustic signals used for the inversion showed peak amplitudes of up to $200 \mathrm{~Pa}$ within a few hundred metres distance from the source. Our results indicate dipole vectors ranging up to $10^{8} \mathrm{~kg} \mathrm{~m} \mathrm{~s}^{-2}$. Although the Tungurahua stations recorded 200 Pa peak at $5 \mathrm{~km}$ distance from the vent, the resulting dipole strengths are comparable to those of Mt Erubus. Because the dipole strength at Mt Erubus was calculated using a dipole-only model, the result may have been overestimated by incorporating part of the monopole into the dipole radiation, as Johnson et al. (2008) noted. This may explain the similarity between dipole strengths estimated for Tungurahua and Mt Erebus, although infrasound signals at Tungurahua volcano show much stronger amplitudes.

It should be noted that modelling presented here only accounts for the horizontal component of an arbitrary dipole in a half-space. The original dipole may include a vertical component, but it cannot be estimated with the present station configuration, as shown in Section 3. The real dipole may therefore be stronger than the estimated horizontal results reported here.

Since monopole, dipole and quadrupole source models of volcanic infrasound have all been proposed (Woulff \& McGetchin 1976), it is still unclear which acoustic source type dominates during volcanic explosions. A monopole source model was used in studies of Strombolian explosions at Erebus and Karymsky (Lees et al. 2004; Johnson et al. 2004, 2008), a large rockfall at Mt St Helens (Moran et al. 2008), and bubble oscillations at the lava surface at Shishaldin (Vergniolle \& Caplan-Auerbach 2004), Stromboli (Vergniolle \& Brandeis 1994) and Erta Ale (Bouche et al. 2010). Only a few studies have addressed a dipole source model for volcanic explosions (Woulff \& McGetchin 1976; Vergniolle \& CaplanAuerbach 2006; Johnson et al. 2008; Caplan-Auerbach et al. 2010). The acoustic network geometry may be one reason for the lack of dipole modelling; full 3-D radiation patterns for a dipole solution are difficult to record on stations placed on the ground surface.

\section{DISCUSSION}

In general the source of volcanic infrasound is associated with atmospheric vibration in the vicinity of the volcano vent. The acoustic source region is defined here by an artificial boundary that includes the vent (Fig. 7c). By incorporating the vent geometry in the source region, which is still compact compared to the source-receiver distance, equations developed in Sections 2 and 3 can be adapted for source inversion with no modification. Once we consider the source 

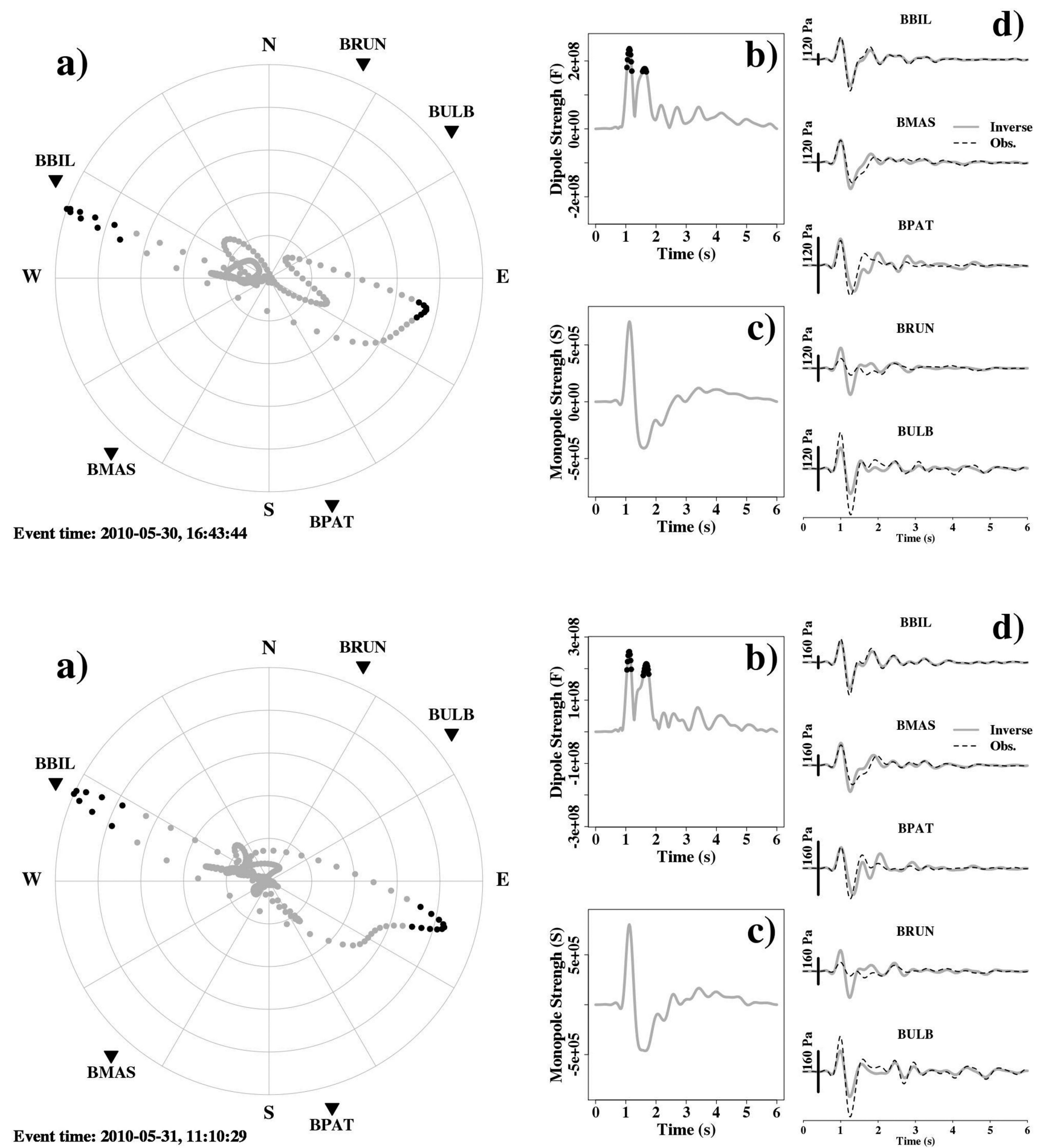

Figure 5. Data fitting and estimated source parameters for infrasound records from Tungurahua. The figure shows examples of two different events. (a) Dipole direction over the source time function is plotted. The magnitude of the dipole is normalized to the maximum value of the source function. Both events show clear WNW directionality of infrasound radiation. Source time functions for (b) a dipole and (c) a monopole are shown. The dipole source function shows consistent directivity before and after the maximum amplitude of pressure (bold black line). (d) The fit of observations and models is shown. The combination of the negative pressure of the dipole and the positive pressure of the monopole produced a small positive amplitude at BPAT. Most stations have acceptably good fits but BRUN exhibits a relatively large discrepancy. This might be attributed to the highly complex topography near the station.

as compact, there is an equivalence between the multipole solutions and physical pressure fluctuations in the source region, similar to the force equivalence of a fault in seismology. In this section, we discuss several possible source mechanisms inside the source region, which are attributed to the inverted acoustic multipoles.

\subsection{Direct sources}

Volcanic explosions and jets (or material flow) are direct sources of infrasounds. Rapid expansion of the compressed gas caused by explosions can be modelled as an acoustic monopole, and represent 
Table 2. Condition number and standard deviations of the source parameters for two events as shown in Fig. 5. $S$ and $|F|$ denote monopole and dipole strengths, respectively. Percentages of the standard deviation with respect to the estimates are given in parentheses.

\begin{tabular}{ccccc}
\hline Event time & Condition no. & $S\left(\mathrm{~kg} \mathrm{~s}^{-1}\right)$ & $|F|\left(\mathrm{kg} \mathrm{m} \mathrm{s}^{-2}\right)$ & Azimuth $\left(^{\circ}\right)$ \\
\hline $2010-5-30,16: 43: 44$ & $3.0 \times 10^{5}$ & $\pm 2.9 \times 10^{4}(4$ per cent $)$ & $\pm 2.1 \times 10^{7}(9$ per cent $)$ & $\pm 3.2^{\circ}$ \\
$2010-5-30,17: 45: 03$ & $3.0 \times 10^{5}$ & $\pm 2.7 \times 10^{4}$ (5 per cent) & $\pm 2.0 \times 10^{7}(9$ per cent $)$ & $\pm 3.5^{\circ}$ \\
\hline
\end{tabular}
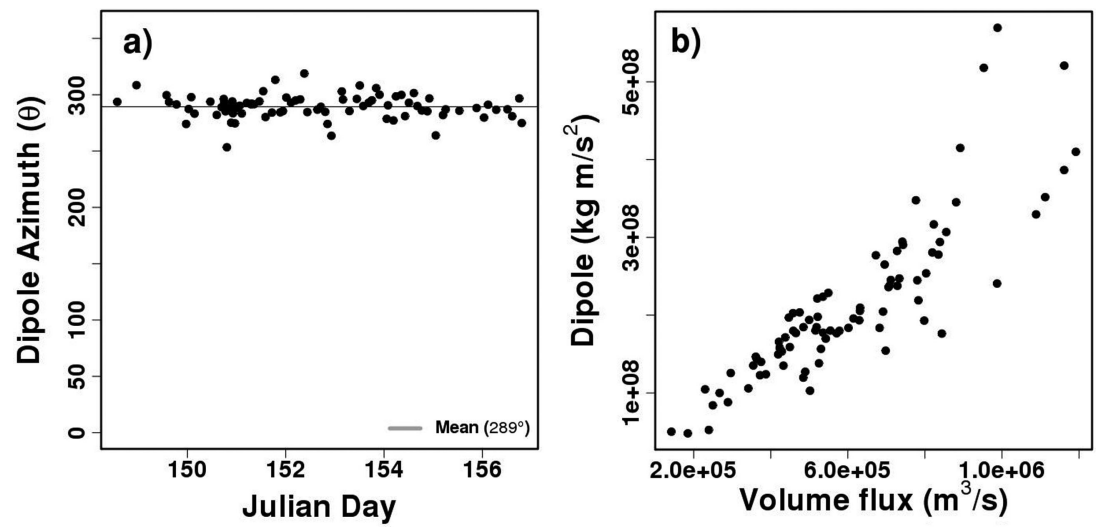

Figure 6. Estimated source parameters using events at Tungurahua volcano. (a) Change of dipole azimuth during the field experiment. (b) Volume flux estimated from monopole and dipole strengths.
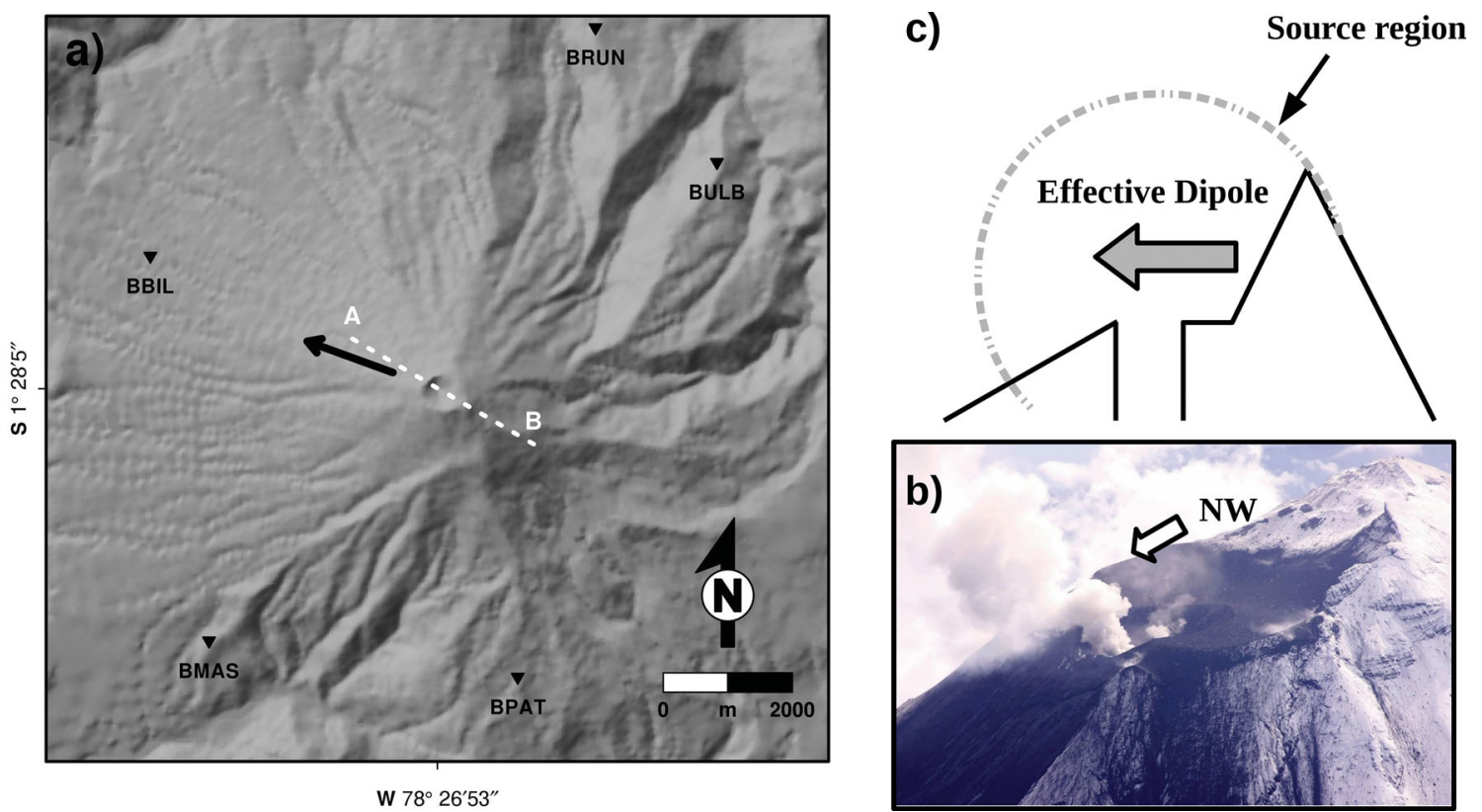

Figure 7. Crater geometry of Tungurahua volcano. (a) The apparent direction of the vent opening (line AB) closely matches the $289^{\circ}$ mean azimuth (black arrow) of the dipole inverted from infrasound observations. (b) Photo of the Tungurahua crater taken on 2011 November 29. The crater is significantly asymmetric with longest diameter $500 \mathrm{~m}$. The SE rim is about $300 \mathrm{~m}$ higher than that of the NW. Photo courtesy of Patricio Ramón (IG). (c) Schematic model of the effective acoustic dipole. The presence of the vent wall probably produces the effective dipole due to the interaction of fluid and sound with the solid boundary (see the text for details).

the dominant source of the observed monopole. Moving objects that subsequently exert forces on the fluid, however, contribute as a dipole source. If the axis of the vent opening is inclined relative to the vertical axis, and materials are ejected in this direction, the resultant effect will be equivalent to a horizontal dipole component. Based on visual observation, the vent opening direction at Tungurahua volcano was not significantly tilted from the vertical. In this case, the vertical flow should be modelled as a vertical dipole, and the observed, strong, horizontal dipole is not accounted for.

\subsection{Diffraction and reflection}

The interaction of sound with solid boundaries inside the source region may account for some of the observed radiation patterns. Theoretical calculations of sound reflection and diffraction using finite difference time domain (FDTD) method (Kim \& Lees 2011) can be compared to multipole approximations. The southeast vent wall at Tungurahua was represented by a semi-circular, $200 \mathrm{~m}$ radial half disc, $200 \mathrm{~m}$ thick (Fig. 8a). Using the mirror image of the disc, sound radiation patterns were computed in the presence of the 

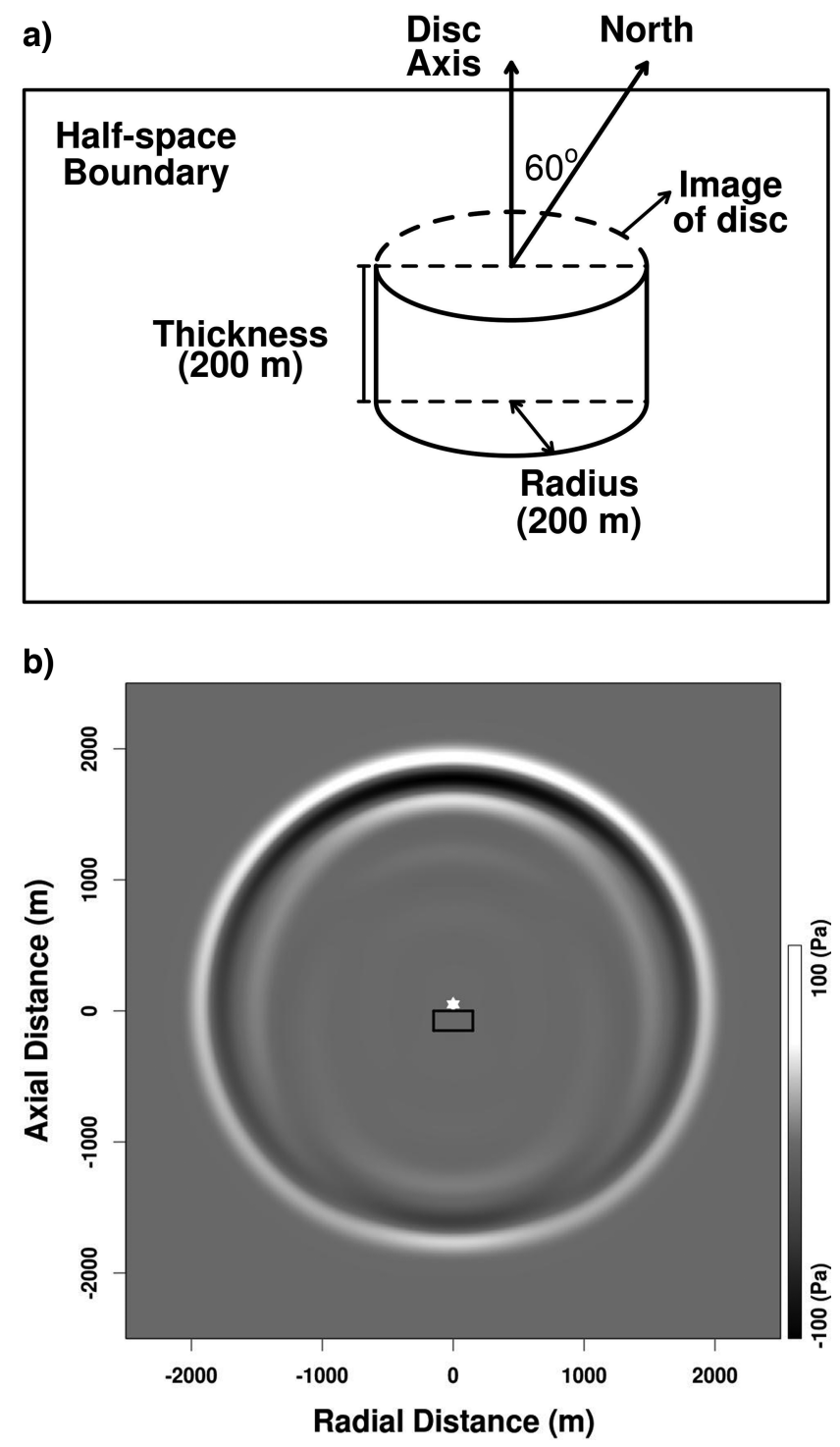

Figure 8. (a) Configuration for FDTD modelling. The rigid half-space was achieved by the image of the disc. (b) Snapshot of sound radiation near the disc. The source was excited by a monopole on the centre of the disc.

half-space. Because of azimuthal symmetry of the computational domain, the 3-D wavefield can be computed efficiently in the cylindrical coordinate (Kim \& Lees 2011). A homogeneous medium (air velocity $=340 \mathrm{~m} \mathrm{~s}^{-1}$ ) was assumed and a monopole source of the Gaussian function ( $1 \mathrm{~Hz}$ corner frequency) was used to excite the sound field. A dipole source was not used as the direct source because there is no evidence of significant horizontal fluid flow discussed in Section 7.1, and vertical dipole does not contribute to the horizontal asymmetric radiation. The frequency was estimated from observed infrasound which showed peak frequencies near 1 $\mathrm{Hz}$, rapidly attenuating at higher frequencies. The source was placed on the disc axis by the wall illustrated in Fig. 8(b). Sound pressures were obtained on the half-space boundary, $3 \mathrm{~km}$ distant from the source in all azimuthal directions, well enough away such that nearfield effects can be ignored. Amplitudes, relative to the maximum amplitude in the propagating sound field, were then assumed to be measures of the effects of reflection and diffraction.

The modelled pressure distribution, compared with the multipole radiation pattern determined by observations, is shown in Fig. 9. The peak-amplitude ratios of each station relative to BBIL station are presented showing a considerable variance. The large variance is probably attributed to either complex source mechanisms which cannot be explained by the combination of the monopole and the dipole or to local noise at the stations. Because the amplitudes are ratios of each station to BBIL, local noise at BBIL has a compounding effect on variance in this plot. Even though the observed amplitudes show such a large variance, the inversion method appears to point to the best-fit solutions including a monopole and dipole.

While the monopole produces an omni-directional radiation, the dipole contributes to the varying amplitude dependent on the azimuthal direction. The dipole produces a positive amplitude of the first arrival in BBIL direction, which constructively interferes with the monopole amplitude. At the same time, a negative pressure of the first arrival is built up by the dipole in the opposite direction near station BPAT. This destructive interference with the positive monopole produces a small, positive amplitude at BPAT. Because the observed dipole is alternating (Fig. 5a), the dipole direction is reversed after the first arrival, giving a large negative amplitude at BBIL and small negative amplitude at BPAT (Fig. 5d) due to interference with the negative monopole (Fig. 5c). Even though the strength of the dipole is larger than the monopole by an order of $\sim 10^{2}$ (Fig. 6), the combined radiation pattern shows positive amplitudes of the first arrival at all five stations. Because a dipole is represented by two alternating, closely spaced, monopole sources, the radiated energy cancels, and is a much less efficient source than a monopole source (Lighthill 1952).

The diffraction (and reflection) shows the characteristic directional pattern presented in Fig. 9. The smallest radiation amplitude does not occur immediately behind the wall, but rather appears slightly off that direction. This is because edge waves diffracted at the edge of the disc are superposed constructively at the axis of the disc, and destructive interference occurs slightly off the axis. In comparison with the multipoles, the directivity of the diffraction is remarkably aligned with it. The diffraction pattern explains well the large amplitude at BBIL and the small amplitude at BBAT. However, it is evident that the degree of directivity of the multipole is larger than expected from the theoretical diffraction pattern. The observed ratio of BPAT to BBIL (median value $\simeq 0.15$ ) is smaller than the value of 0.4 expected from the diffraction alone, suggesting that the amplitude of BBIL is much larger. This discrepancy is too large to be explained by modelling errors associated with the simplified model. Of course, assumptions of a semi-circular backwall and inaccurate wall dimensions may give rise to errors in the obtained diffraction pattern. At low frequency (1 Hz peak frequency, wavelength $=340 \mathrm{~m}$ ), however, variations in wall dimensions of up to several tens of metres will not affect the diffraction radiation patterns significantly. This suggests that the diffraction effects partially influenced observed directivity, although other factors must have a significant impact on the additional variation of directivity.

\subsection{Aerodynamic flow}

In the previous section, we considered interaction of acoustic waves, generated by fluid flow, with solid boundaries in terms of diffraction and reflection. If solid boundaries are present in the source region and vent dimensions cannot be ignored compared to wavelengths, interaction between the material fluid flow (not sound) and solid boundaries may play an important role in sound production (Lighthill 1952). Effects of solid-fluid interactions can be shown to be equivalent to a distribution of dipoles representing the force with 


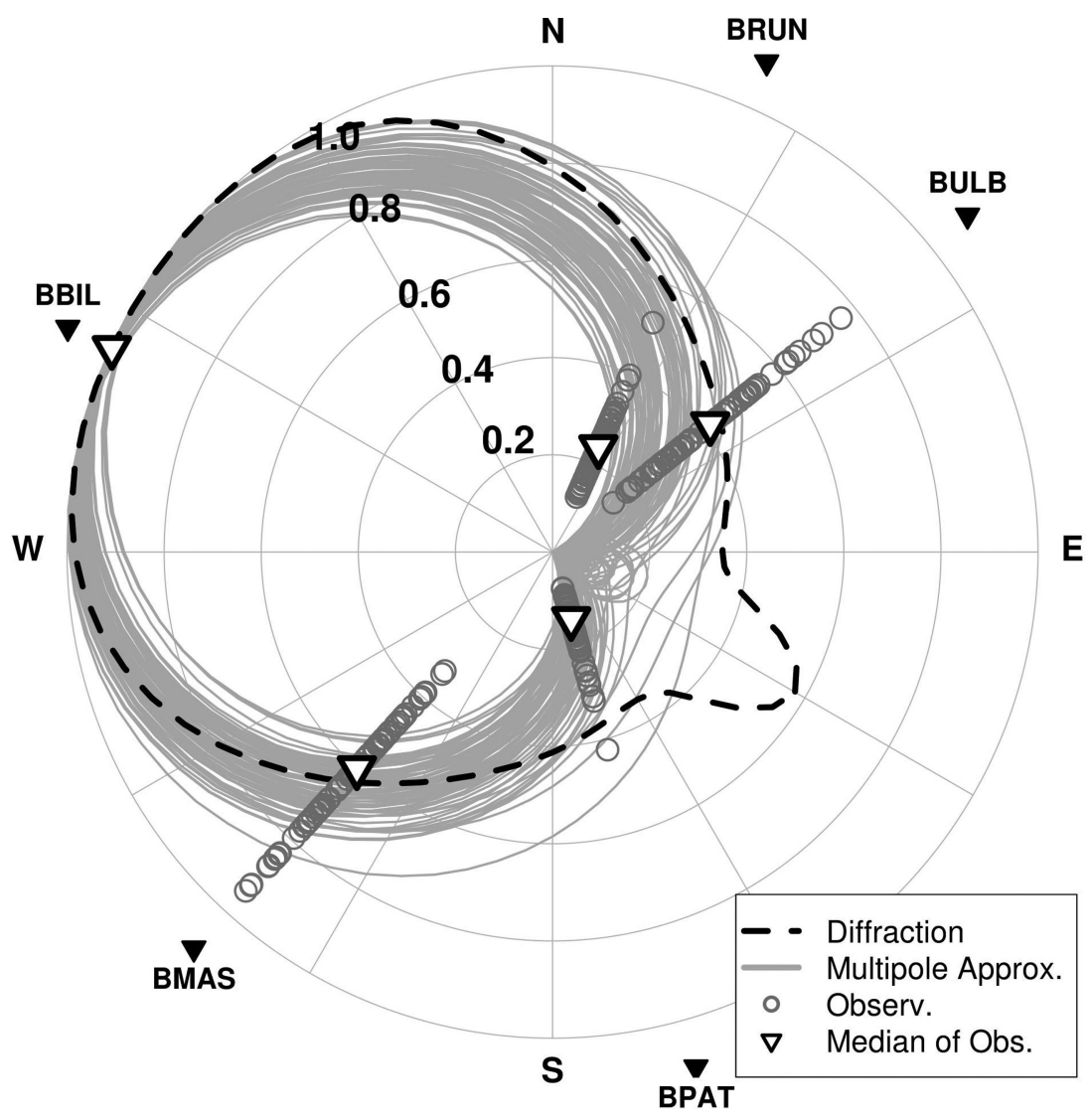

Figure 9. Comparison between the diffraction pattern (dot line) and the multipole approximation (grey solid lines). All curves are normalized to maximum values. Observed pressures (circles) at stations are overplotted, and median values are indicated by invert triangles. The directivity of the diffraction is well aligned with the multipole approximation, but the small amplitude of BPAT cannot be explained solely by the diffraction (see the text for details).

which the solid boundary acts upon the fluid (Curle 1955; Leehey \& Hanson 1970). In volcanology, sound emitted by a steady gas jet carrying solid fragments has been previously accounted for by a dipole (Woulff \& McGetchin 1976; Vergniolle \& Caplan-Auerbach 2006). The earlier studies considered the solid-fluid interaction (gas jet and solid particles or gas jet and solid wall) to be the sources of the dipole. In our study, explosion sources are dominant in the observed signals as opposed to steady gas jetting observed earlier. Rapid expansion of gas probably interacts with the vent wall, exerting significant forces on the flow. This exerting force should behave as an acoustic dipole. Because the vent wall of Tungurahua is asymmetric, the induced dipole is aligned with the normal to the wall surface.

We have proposed three possible source mechanisms based on observations and numerical modelling. The multipole source, inverted from the observations, showed a strong dipole component, significantly stronger than that expected from the diffraction modelling. The source physics that explains the multipole radiation is very likely to be non-unique because the multipole analysis calculates approximated acoustic sources that are equivalent to the source physics occurring during volcanic explosions. Different source mechanisms can obviously generate the same radiation pattern. It is important to note that sound diffraction by and fluid interaction with the asymmetric vent wall are both possible origins of the observed strong dipole radiation pattern. In both cases, the vent wall plays a critical role in developing the observed dipole radiation. The amount of mass outflux accompanied by explosions can be approximated by the strength of monopole component, if we assume the dipole component is produced by the effects of the wall. Even if the interaction with the vent wall does not exactly match the predicted dipole, this approximation should be useful in practice, especially when the number of stations in volcanic infrasound networks is limited.

\section{CONCLUSION}

We have derived approximate solutions for acoustic radiation in a rigid half-space and shown how to calculate the infrasound-acoustic response of waves emanating from volcanic explosions. The result indicates that a vertical dipole does not contribute to pressure disturbances near the half-space boundary (the Earth's surface) and inversion using this assumption should be treated with caution. A reliable inversion procedure was presented for estimating mass outflow and force generated during volcanic explosions. Monopole and dipole sources were simultaneously inverted for, and stable acoustic source parameters were extracted using at least three stations. The method was applied to observations at Tungurahua volcano in Ecuador, where mass outflux and dipole strength exhibited good agreement with previous estimates at other locations around the globe. We relate the acoustic amplitude directivity of explosions at Tungurahua to vent geometry. The approach taken was completely general and will be applicable to in other volcanic settings where extracting parameters of source dynamics are critical.

\section{ACKNOWLEDGMENTS}

This research was supported by the National Science Foundation Grant EAR0738768. We thank Hiroyuki Kumagai for establishing 
the high-quality seismo-acoustic network at Tungurahua through the Japanese JICA program. We also thank the authorities and staff of the Instituto Geofisico and Observatotio Vulcanologico del Tungurahua for sharing data and providing support.

\section{REFERENCES}

Bouche, E., Vergniolle, S., Staudacher, T., Nercessian, A., Delmont, J.-C., Frogneux, M., Cartault, F. \& Pichon, A.L., 2010. The role of large bubbles detected from acoustic measurements on the dynamics of Erta 'ale lava lake (Ethiopia), Earth planet. Sci. Lett., 295(1-2), 37-48.

Caplan-Auerbach, J., Bellesiles, A. \& Fernandes, J.K., 2010. Estimates of eruption velocity and plume height from infrasonic recordings of the 2006 eruption of Augustine volcano, Alaska, J. Volc. Geotherm. Res., 189(1-2), $12-18$.

Cheney, E. \& Kincaid, D., 2007. Numerical Mathematics and Computing, Brooks/Cole Pub. Co., Pacific Grove, CA

Curle, N., 1955. The influence of solid boundaries upon aerodynamic sound, Proc. R. Soc. Lond. A, Math. Phys. Sci., 231(1187), 505-514.

Fee, D., Garces, M. \& Steffke, A., 2010. Infrasound from Tungurahua volcano 2006-2008: Strombolian to Plinian eruptive activity, J. Volc. Geotherm. Res., 193(1-2), 67-81.

Garces, M.A. \& McNutt, S.R., 1997. Theory of the airborne sound field generated in a resonant magma conduit, J. Volc. Geotherm. Res, 78(3-4), $155-178$.

de Groot-Hedlin, C., 2008. Finite-difference time-domain synthesis of infrasound propagation through an absorbing atmosphere, J. acoust. Soc. Am., 124(3), 1430-1441.

Jackson, D.D., 1972. Interpretation of inaccurate, insufficient and inconsistent data, Geophys. J. R. astr. Soc., 28(2), 97-109.

Johnson, J., 2007. On the relation between infrasound, seismicity, and small pyroclastic explosions at Karymsky volcano, J. geophys. Res., 112, B08203, doi:10.1029/2006JB004654.

Johnson, J., Aster, R., Jones, K., Kyle, P. \& McIntosh, B., 2008. Acoustic source characterization of impulsive Strombolian eruptions from the Mount Erebus lava lake, J. Volc. Geotherm. Res., 177(3), 673-686.

Johnson, J.B. \& Lees, J.M., 2010. Sound produced by the rapidly inflating Santiaguito Lava Dome, Guatemala, Geophys. Res. Lett., 37, L22305, doi:10.1029/2010GL045217.

Johnson, J.B., Aster, R.C. \& Kyle, P.R., 2004. Volcanic eruptions observed with infrasound, Geophys. Res. Lett., 31(14), L14604, doi:10.1029/2004GL020020.

Kim, K. \& Lees, J.M., 2011. Finite-difference time-domain modeling of transient infrasonic wavefields excited by volcanic explosions, Geophys. Res. Lett., 38(6), L06804, doi:10.1029/2010GL046615.

Leehey, P. \& Hanson, C., 1970. Aeolian tones associated with resonant vibration, J. Sound Vib., 13(4), 465-483.

Lees, J., Gordeev, E. \& Ripepe, M., 2004. Explosions and periodic tremor at Karymsky volcano, Kamchatka, Russia, Geophys. J. Int., 158(3), 1151-1167.

Li, K.M., Taherzadeh, S. \& Attenborough, K., 1997. Sound propagation from a dipole source near an impedance plane, J. acoust. Soc. Am., 101(6), 3343-3352.

Lighthill, M.J., 1952. On sound generated aerodynamically. I. General theory, Proc. R. Soc. London. A, Math. Phys. Sci., 211(1107), 564-587.

Lighthill, M.J., 1978. Waves in Fluids, Cambridge University Press, New York, NY.

Matoza, R., Fee, D., Garcés, M., Seiner, J., Ramón, P. \& Hedlin, M., 2009. Infrasonic jet noise from volcanic eruptions, Geophys. Res. Lett., 36(8), L08303, doi:10.1029/2008GL036486.

Moran, S.C., Matoza, R.S., Garcs, M.A., Hedlin, M.A.H., Bowers, D., Scott, W.E., Sherrod, D.R. \& Vallance, J.W., 2008. Seismic and acoustic record- ings of an unusually large rockfall at Mount St. Helens, Washington, Geophys. Res. Lett., 35(19), L19302, doi:10.1029/2008GL035176.

Morse, P. \& Ingard, K., 1986. Theoretical acoustics, Princeton University Press, Princeton, NJ.

Ostashev, V., Wilson, D., Liu, L., Aldridge, D., Symons, N. \& Marlin, D., 2005. Equations for finite-difference, time-domain simulation of sound propagation in moving inhomogeneous media and numerical implementation, J. Acoustic. Soc. Am., 117, 503.

Ozerov, A., Ispolatov, I. \& Lees, J., 2003. Modeling strombolian eruptions of karymsky volcano, kamchatka, russia, J. Volc. Geotherm. Res, 122(3-4), 265-280.

Parker, R., 1994. Geophysical Inverse Theory, Princeton University Press, Princeton, NJ.

Pierce, A., 1989. Acoustics: An Introduction to its Physical Principles and Applications, Acoustical Society of America, Woodbury, NY.

Ripepe, M., Marchetti, E. \& Ulivieri, G., 2007. Infrasonic monitoring at Stromboli volcano during the 2003 effusive eruption: insights on the explosive and degassing process of an open conduit system, J. geophys. Res., 112(B9), B09207, doi:10.1029/2006JB004613.

Rossing, T., 2007. Springer Handbook of Acoustics, Springer Verlag, New York, NY.

Ruiz, M., Lees, J. \& Johnson, J., 2006. Source constraints of Tungurahua volcano explosion events, Bull. Volcanol., 68(5), 480-490.

Stump, B.W. \& Johnson, L.R., 1977. The determination of source properties by the linear inversion of seismograms, Bull. seism. Soc. Am., 67(6), $1489-1502$.

Sutherland, L.C. \& Bass, H.E., 2004. Atmospheric absorption in the atmosphere up to $160 \mathrm{~km}$, J. acoust. Soc. Am., 115(3), 1012-1032.

Vergniolle, S. \& Brandeis, G., 1994. Origin of the sound generated by Strombolian explosions, Geophys. Res. Lett., 21(18), 1959-1962.

Vergniolle, S. \& Caplan-Auerbach, J., 2004. Acoustic measurements of the 1999 basaltic eruption of Shishaldin volcano, Alaska: 2. Precursor to the subplinian phase, J. Volc. Geotherm. Res., 137(1-3), 135-151.

Vergniolle, S. \& Caplan-Auerbach, J., 2006. Basaltic thermals and subplinian plumes: constraints from acoustic measurements at Shishaldin volcano, Alaska, Bull. Volcanol., 68, 611-630.

Vergniolle, S., Brandeis, G. \& Mareschal, J., 1996. Strombolian explosions 2. Eruption dynamics determined from acoustic measurements, J. geophys. Res., 101(B9), 20 449-20 466.

Vergniolle, S., Boichu, M. \& Caplan-Auerbach, J., 2004. Acoustic measurements of the 1999 basaltic eruption of Shishaldin volcano, Alaska 1. Origin of Strombolian activity, J. Volc. Geotherm. Res., 137(1-3), 109-134.

Woulff, G. \& McGetchin, T.R., 1976. Acoustic noise from volcanoes: theory and experiment, Geophys. J. R. astr. Soc., 45, 601-616.

\section{SUPPORTING INFORMATION}

Additional Supporting Information may be found in the online version of this article:

Supplement. Data fitting and estimated source parameters for 'acoustic noise' which was not related to volcanic activities. Two examples are shown for different periods. (a) Dipole direction, (b) dipole strength, (c) monopole strength and (d) data fitting are plotted. Dipole directions from the noise were inconsistent with the $289^{\circ}$ azimuth determined from the volcanic source and indicated which stations showed the highest noise level at that time.

Please note: Wiley-Blackwell are not responsible for the content or functionality of any supporting materials supplied by the authors. Any queries (other than missing material) should be directed to the corresponding author for the article. 